\title{
Male Breast Carcinoma Metastatic to the Choroid: A Case Report and Opinions of Management
}

\author{
Weida Fu*, Shurong Zheng*, Jieyu Zhou (D, Kangkang Lu, Yiqiao Lu, Guilong Guo (D) \\ Department of Breast Surgery, The First Affiliated Hospital of Wenzhou Medical University, Wenzhou, Zhejiang, People's Republic of China \\ *These authors contributed equally to this work \\ Correspondence: Guilong Guo, Department of Breast Surgery, The First Affiliated Hospital of Wenzhou Medical University, Wenzhou, Zhejiang, \\ People's Republic of China, Tel/Fax +86-I386770936I, Email guoguilong@sina.com
}

\begin{abstract}
Male breast carcinoma metastatic to the choroid is very rare and often related to poor prognosis. Herein, we report the findings in a Chinese male breast cancer patient who developed choroidal metastasis, and give opinions on systemic treatments. A 45-year-old Chinese male represented with difficulty breathing and visual impairment in the left eye 6 years after his breast cancer surgery and postoperative adjuvant treatment. PET/CT revealed multi-organs metastasis of the patient. The IHC indicated the lung lesion to be originated from the breast (ER+/PR+/HER2-). Eye examination provided evidence for breast cancer choroidal metastasis. Two cycles of TX (docetaxel + capecitabine) followed by two courses of GP (gemcitabine + cis-platinum) were applied as salvage chemotherapy. Metastases in his lung and bone remained stable. As for choroidal metastasis, a regimen of CDK4/6 inhibitor (Palbociclib) plus fulvestrant was recommended to the patient, which led to a good response. Notably, CDK4/6 inhibitor combined with endocrine therapy may be considered as an effective treatment for hormonal receptor-positive breast cancer patients with choroidal metastasis. We recommend that eye examination should not be neglected in breast cancer patients.
\end{abstract}

Keywords: choroidal metastasis, male breast cancer, systemic therapy, CDK4/6 inhibitor, case report

\section{Introduction}

Male breast cancer is infrequent and represents approximately $1 \%$ of all breast cancers (BC). Although $\mathrm{BC}$ in men and in women is similar in some ways, male $\mathrm{BC}$ has distinct features. The mean age at diagnosis of $\mathrm{BC}$ is five years later in men than in women. Male $\mathrm{BC}$ is more frequently hormone receptor-positive and more likely to carry mutations in DNA damage repair genes. Among other risk factors concerning male $\mathrm{BC}$ are black race, radiation exposure, use of exogenous estrogen and hormonal associated diseases like Klinefelter's syndrome. ${ }^{1}$

Choroidal metastasis (CM) is the most frequent ocular tumor. The most frequent primary tumors in CM are located in the mammary glands and the lung. ${ }^{2} \mathrm{CM}$ usually occurs 3 to 5 years after $\mathrm{BC}$ diagnosis, mostly are female and the majority are on the left. ${ }^{3} \mathrm{CM}$ secondary to male $\mathrm{BC}$ is rarely reported. The precise incidence of $\mathrm{CM}$ in male $\mathrm{BC}$ is unknown, but it has been estimated that in the United States, about 25 cases of male BC metastasize to the choroid every year. ${ }^{4}$ The treatment strategy for $\mathrm{CM}$ in male $\mathrm{BC}$ has not yet been established and there are no reports on the efficacy of CDK4/6 inhibitor yet. In this paper, we report a case of male BC metastatic to the choroid, in which the patient received CDK4/6 inhibitor plus fulvestrant treatment, with a favorable outcome observed.

\section{Case Presentation}

A 39-year-old man was admitted with a 3-year medical history of a lump in his left breast in 2012. He was a non-smoker and denied a history of alcohol intake. He had no history of hypertension or diabetes or hepatitis B. There was no family 
history of $\mathrm{BC}$, either. He was treated with radical mastectomy with lymphadenectomy. The postoperative pathological report indicated invasive ductal $\mathrm{BC}$ on the left side and without axillary lymphatic metastasis in 12 lymph nodes. The tumor mass was $1.5 \mathrm{~cm}$ in diameter and the TNM stage was diagnosed with pT1cN0M0 Stage I. Immunohistochemistry (IHC) result showed the tumor was estrogen receptor (ER)-positive, progesterone receptor (PR)-positive, human epidermal growth factor receptor 2 (HER2)-negative and Ki67 (about 15\%+) (Figure 1). Then, the patient received ECF chemotherapy (Epirubicin $80 \mathrm{mg}$, Cyclophosphamide $800 \mathrm{mg}$, 5-Fluorouracil $750 \mathrm{mg}$ ) in six cycles, followed by adjuvant anastrozole for 5 years.

Approximately one year later, a workup using chest computed tomography (CT) (Figure 2A) for irritating cough and difficulty breathing revealed a lung mass. A fiberoptic bronchoscopic biopsy was performed, and IHC indicated that ER (+++) almost $90 \%$ strong positive cells, PR (++) almost $70 \%$ strong positive cells, HER2 (-), Ki67 $(40 \%+)$ (Figure 3). The lesion was further confirmed to be adenocarcinoma, consistent with metastasis from the breast. The positron emission tomography/computed tomography (PET/CT) imaging was then performed and showed multiple systemic metastases (Figure 4). In consultation with the severe symptoms, the patient was commenced on salvage chemotherapy with TX (docetaxel $75 \mathrm{mg} / \mathrm{m}^{2}$ D1 and capecitabine $1000 \mathrm{mg} / \mathrm{m}^{2}$ bid D1-14) for two cycles in combination with zoledronic acid $(4 \mathrm{mg} \mathrm{q} 4 \mathrm{w})$. His respiratory symptoms improved markedly during 12-week follow-up, however, the size of the lung lesion still remained stable (Figure 2B). Then, two courses of GP (gemcitabine $1000 \mathrm{mg} / \mathrm{m}^{2} \mathrm{D} 1$, D8 + cis-platinum $25 \mathrm{mg} / \mathrm{m}^{2}$ D1-3) chemotherapy combined with zoledronic acid ( $4 \mathrm{mg} \mathrm{q} 4 \mathrm{w}$ ) was applied. However, he did not achieve any shrinking of the tumor except severe leukopenia (Figure 2C). And during the second course of GP chemotherapy, the patient concurrently reported a gradual reduction in vision in the left eye over 10 days, and was referred to the ophthalmology department for evaluation and management.

Dilated fundus examination showed that in each eye, a round relatively bump yellow-white choroidal mass was noted to be superior to the optic disc, surrounded by serous leakage (Figure 5A and B). The lesion was larger in the left eye, resulting in disturbing symptoms. Optical coherence tomography (OCT) indicates limited detachment of the retinal neuroepithelial layer in the left eye, associated with subretinal fluid (SRF). In the right eye, localized choroidal swelling

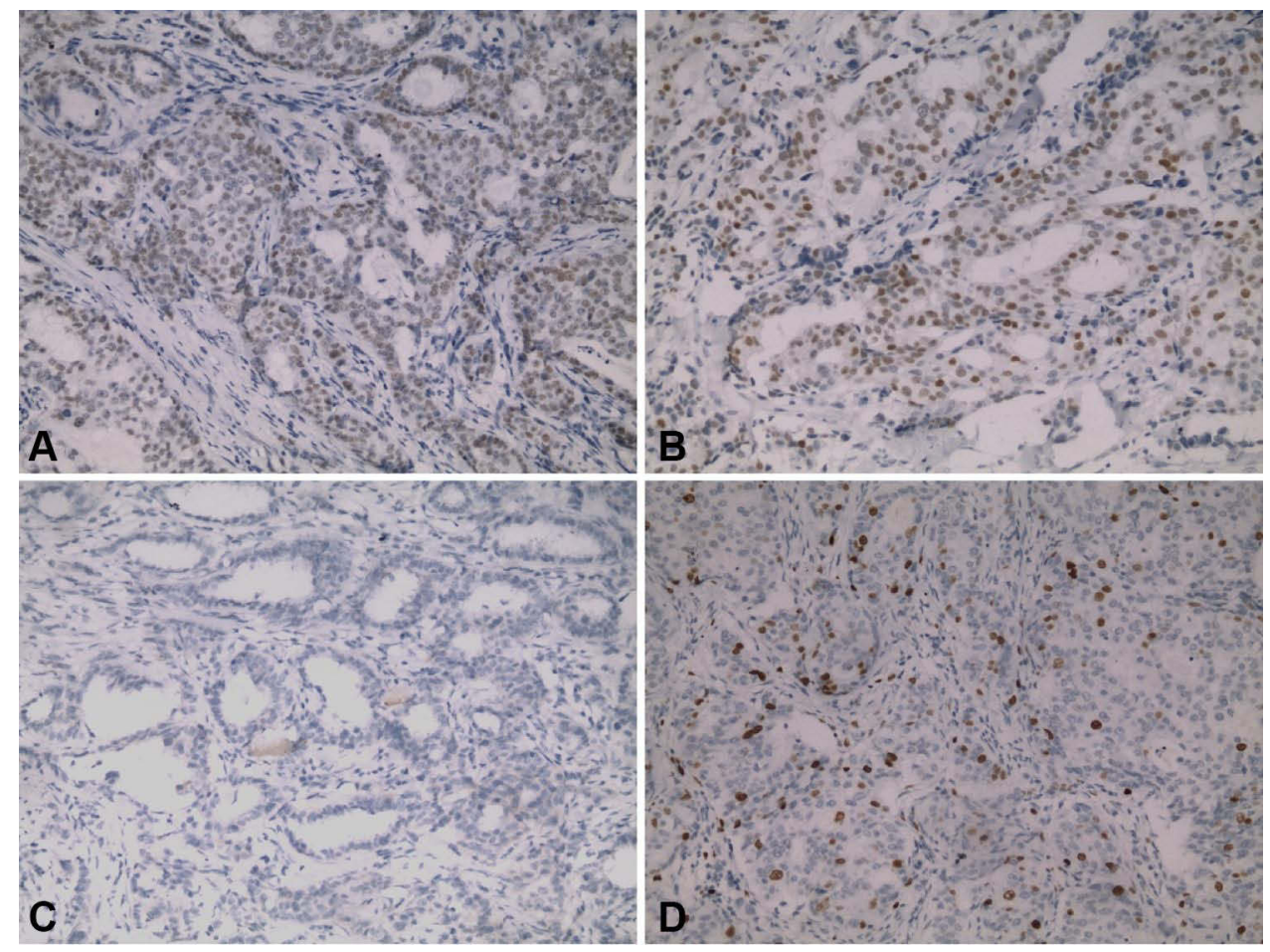

Figure I IHC result of breast carcinoma showed ER (+), PR (+), HER-2 (-), Ki67 (about 15\%+). (A) ER, (B) PR, (C) HER2, (D) Ki67 (x 200). 

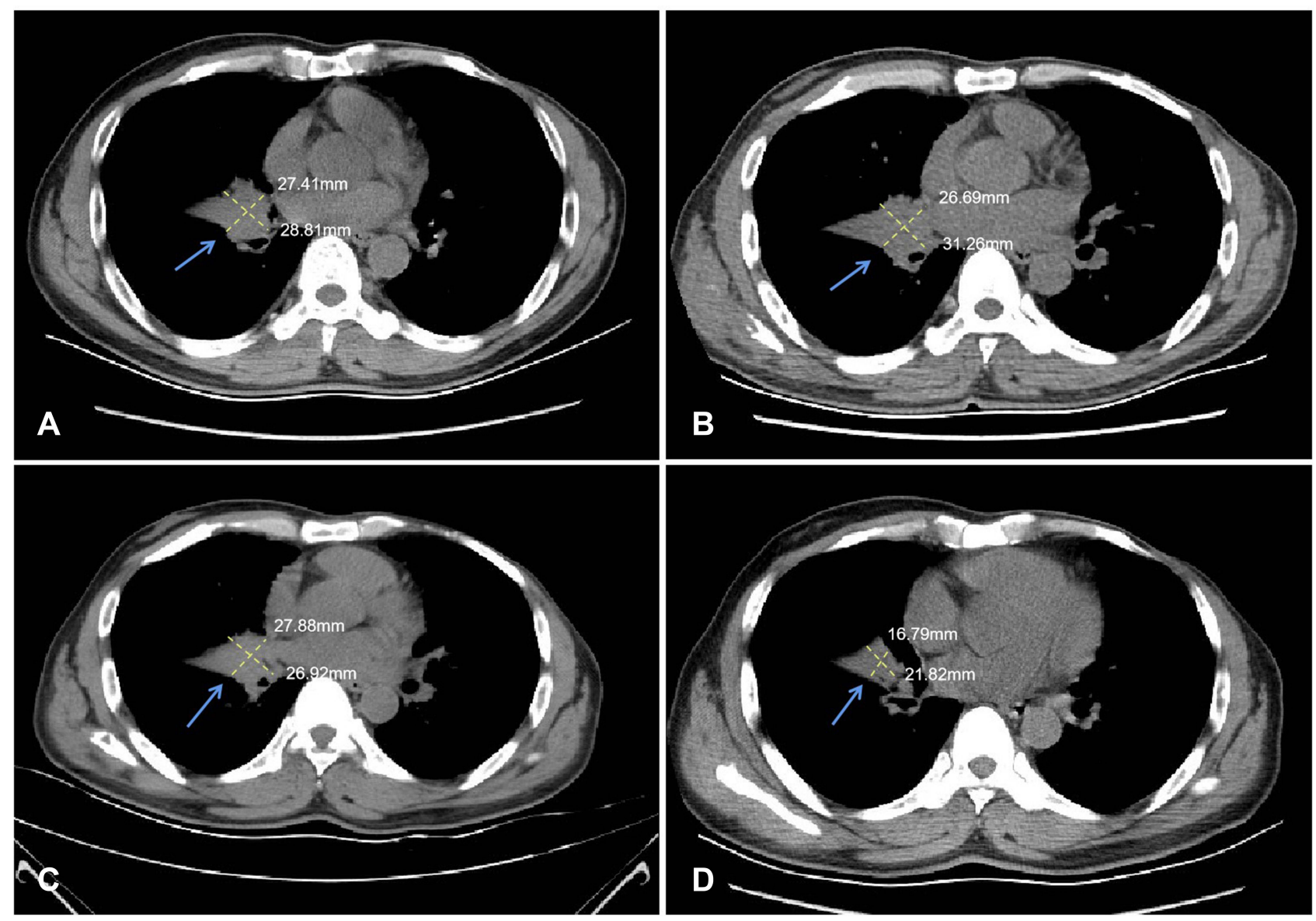

Figure 2 (A) Six years after his radical mastectomy, chest CT indicated a mass with a size of about $27.41 * 28.81 \mathrm{~mm}$ in the right lung. With the completion of (B) two cycles of TX (docetaxel + capecitabine) and (C) two course of GP (gemcitabine + cis-platinum), the size of the lung lesion remained stable. The size of the lesion was about $26.69 * 31.26 \mathrm{~mm}$ in (B) and was about $27.88 * 26.92 \mathrm{~mm}$ in (C). (D) The lesion shrinked slightly after treatment of fulvestrant and CDK4/6 inhibitor, which was about $16.79 * 21.82 \mathrm{~mm}$ in size. The lesions were annotated with blue arrows.

was visible (Figure 6A and B). Genetic test for hereditary breast cancer was conducted, and he had a germline MUTYH mutation (c.74G>A (p.Gly25Asp, Het) and c.53C $>$ T (p.Pro18Leu, Het)) and BRCA1 mutation (c.2048A $>$ G (p. Lys683Arg, Het)). The patient was clinically diagnosed with breast carcinoma metastatic to choroid. The patient refused radiotherapy. Combined treatment with fulvestrant and cyclin-dependent kinases 4 and 6 (CDK4/6) inhibitor (Palbociclib) and gonadotropin-releasing hormone analogue (goserelin) plus zoledronic acid (4 mg q4w) was planned since January 31, 2019. Then, he was closely followed up every 2 months. Surprisingly, chest CT showed the lung lesion shrinked slightly (Figure 2D). Spine CT showed bone lesion remained stable (Figure 7). Examination of the eyes demonstrated the shrinking of choroidal infiltration and the reattachment of the retina (Figures 5C and D, 6C and D). During two-year follow-up, the lesions in his bone and lung remained stable, and the visual field defect improved slightly. So far, this patient is still using the CDK4/6 inhibitor and fulvestrant.

\section{Discussion and Conclusion}

$\mathrm{BC}$ is the most common cancer to affect women in China. ${ }^{5}$ In 2020 , data from GLOBOCAN showed that for the first time, $\mathrm{BC}$ has become the most commonly diagnosed cancer (11.7\% of total cases), surpassing lung cancer $(11.4 \%){ }^{6}$

Despite the low incidence of male breast cancer, the biological characteristics of male $\mathrm{BC}$ and female $\mathrm{BC}$ are not exactly the same. The lifetime risk of male BC is approximately 1:1000, while it is nearly $1: 8$ for a woman. ${ }^{7}$ Male $\mathrm{BC}$ usually expresses ER, PR and androgen receptor (AR); is hormonally responsive; and most commonly present as 


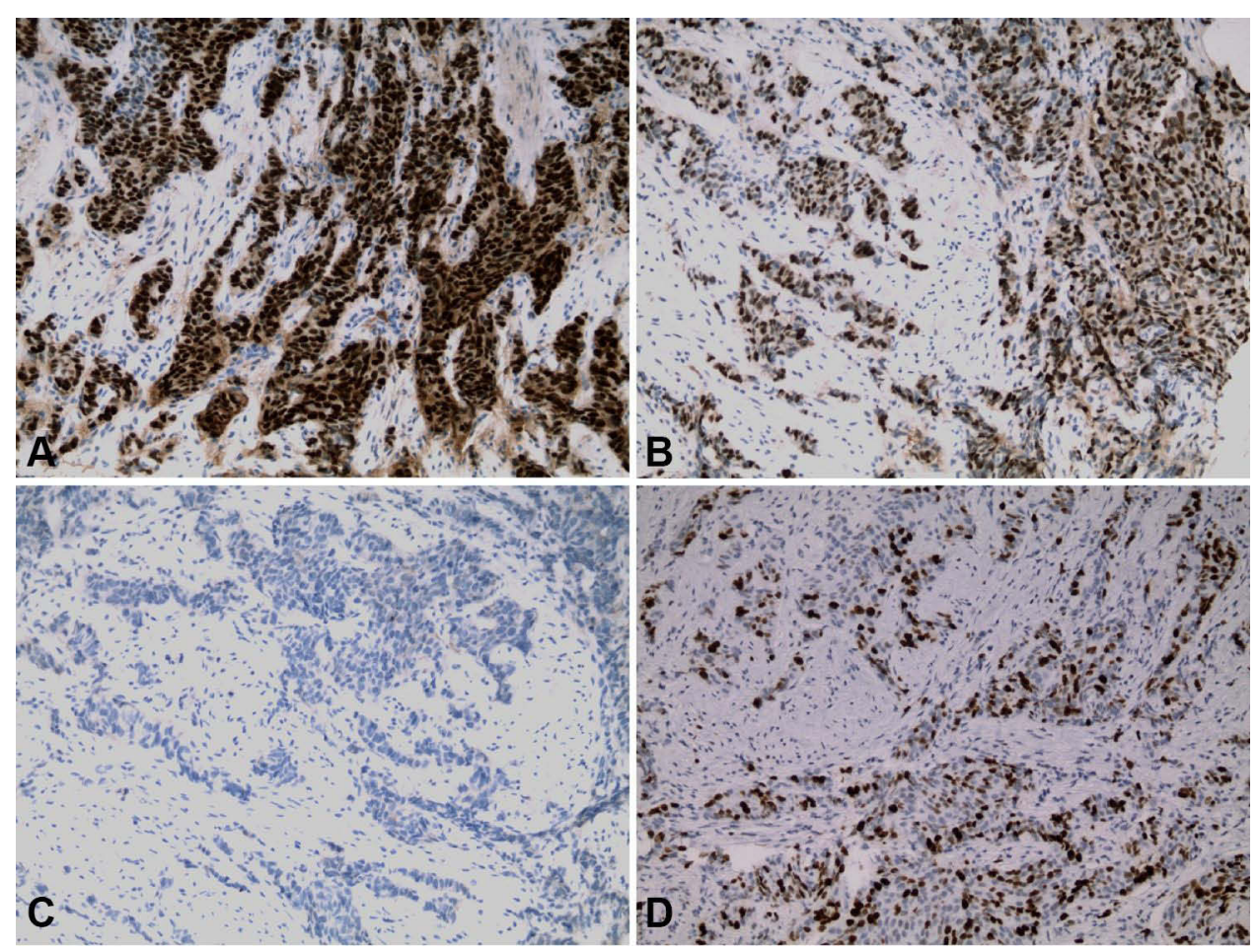

Figure $3 \mathrm{IHC}$ result of pulmonary lesion showed ER (+++) almost 90\% strong positive cells, PR (++) almost 70\% strong positive cells, HER2 (-), Ki67 (40\%+). (A) ER, (B) PR, (C) HER2, (D) Ki67 ( $\times 200)$.

unilateral tumors. ${ }^{8}$ Estimates of the lifetime risk of developing male BC range from $1 \%$ to $5 \%$ for BRCA1 mutation carriers and $5 \%$ to $10 \%$ for BRCA2 mutation carriers, compared to $0.1 \%$ in the general population. ${ }^{9}$ With regard to the comparison of prognosis between male $\mathrm{BC}$ and female $\mathrm{BC}$, conflicting data have been reported. Several studies have indicated that the overall survival (OS) estimates for male breast cancer are significantly lower than for female breast cancer. ${ }^{10-12}$ However, other studies with larger sample sizes have shown that male BC has a better prognosis than female $\mathrm{BC}$ after adjusted for age, treatment, and stage..$^{13,14}$

In recent years, with the drastic improvement of treatment methods, the survival rate of $\mathrm{BC}$ continues to rise. ${ }^{15}$ Unfortunately, some patients diagnosed at an early stage may still develop locoregional or distant tumor recurrence. ${ }^{16}$ Tumor metastasis to the eye is often related to a poor prognosis. Haidar et al indicated that the median survival after $\mathrm{CM}$ in patients with stage I/II BC was 28.7 months, while the median survival of patients with stage III/IV BC was 4.6 months. ${ }^{17}$ An analysis of 1310 eyes in 1111 patients with uveal metastases revealed $90 \%$ involving the choroid. ${ }^{3}$ This may be because the choroid receives $80-85 \%$ of the blood flow in the eye, making it easier for tumor cells to transport. ${ }^{18} \mathrm{BC}$ is the most common carcinoma leading to $\mathrm{CM}$, accounting for $40 \%$ to $54 \%$, followed by lung cancer ( $14 \%$ to $30 \%$ ). The symptoms of $\mathrm{CM}$ are blurred vision (55-70\%), phosphenes and floaters (12\%), visual field defects (15.5\%). Patients mean age at ocular metastases diagnosis is 60 years, those whose primary site is breast are diagnosed at a younger age (57 years). ${ }^{19}$ Our male patient presented with visual field defects in left eye at 45 years, 6 years after his breast cancer surgery.

Nowadays, the mostly used clinical examination methods include ophthalmoscope, OCT, ocular ultrasound, eye CT/MRI, indocyanine green fundus angiography (IC-GA) and ocular metastasis biopsy. Ophthalmoscope is often the first choice as the most convenient and routine fundus examination. CM generally appear as characteristic homogenous, creamy white or yellow masses. Their appearance can be either flat or plateau shaped. ${ }^{20}$ OCT revealed a significant feature of CM, that is so-called the lumpy bumpy lesion. Besides, SRF is another sign related to $\mathrm{CM}$, which can be seen in $67-95 \%$ of cases. Due to its capability to monitor changes in retinal 


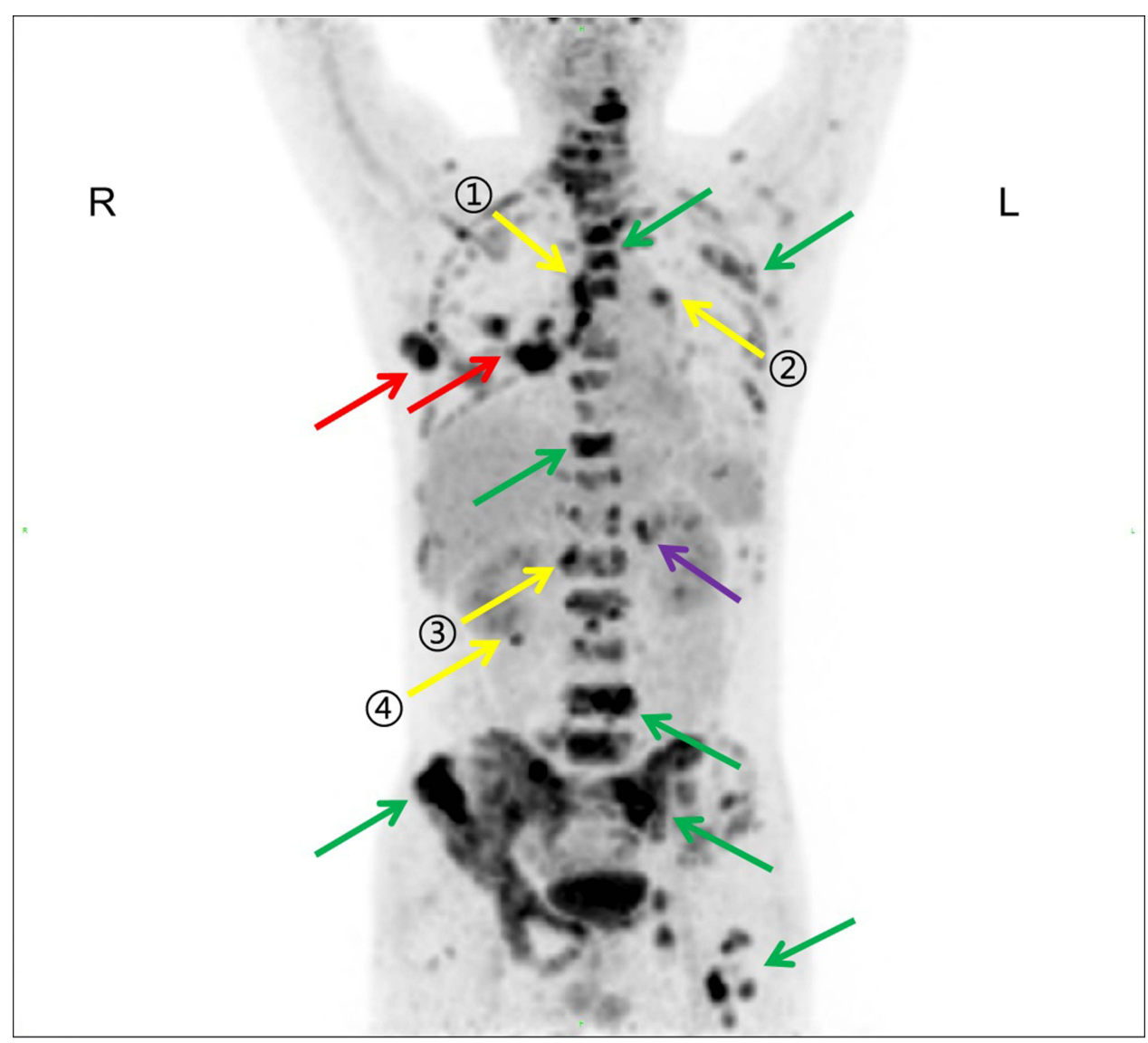

Figure $4 \mathrm{PET} / \mathrm{CT}$ result indicated multiple systemic metastases, including bone (green arrows), lung (red arrows), left adrenal glands (purple arrow), multiple lymph node metastases in the mediastinum (yellow arrow I), hilar (yellow arrow 2), abdominal aorta (yellow arrow 3) and right posterior renal space (yellow arrow 4).

thickness and SRF density, OCT is useful in the follow-up of CM after therapy. ${ }^{21}$ Although tumor biopsy is the gold standard for tumor diagnosis, it is beyond agreement when applied to CM. Some argue that a tumor biopsy carries a risk of tumor cell spreading, and local complications including intravitreal hemorrhage or retinal detachment. Biopsy requires the lesion $>3 \mathrm{~mm}$ in thickness so that it can provide sufficient information. ${ }^{22}$ In other words, it is unnecessary to perform an eye biopsy in a patient with multi-organ metastases if the primary tumor is known. Therefore, we choose to clarify the diagnosis of our patient based on the previous breast cancer history, ophthalmoscope and OCT examination.

Treatments for patient without life-threatening metastases can be limited to the eye, which includes radiotherapy, laser therapy, photodynamic therapy (PDT), intraocular injection of anti-vascular endothelial growth factor (VEGF). ${ }^{23}$ However, systemic therapy is more widely used since most patients suffer from the primary tumor. With regard to $\mathrm{BC}$, the conclusion made by Parrozzani et al suggested that $\mathrm{CMs}$ were more likely to originate from hormonal receptor (HR) positive types. ${ }^{24}$ Therefore, endocrine therapy is considered effective. Manquez et al treated 17 ER and PR positive patients with aromatase inhibitor (AI), 10 patients had regression of both systemic and CM over a mean of 20 months of follow-up. ${ }^{25}$ Demirci et al reported $85 \mathrm{BC}$ patients with $\mathrm{CM}$, who underwent systemic chemotherapy and/or hormone therapy, 52 (65\%) patients achieved regression of CM, 12 (16\%) maintained stability (mean follow-up 10 months). ${ }^{26}$ The patient we reported with ER/PR positive status was classified as luminal A, which was consistent with previous findings. 


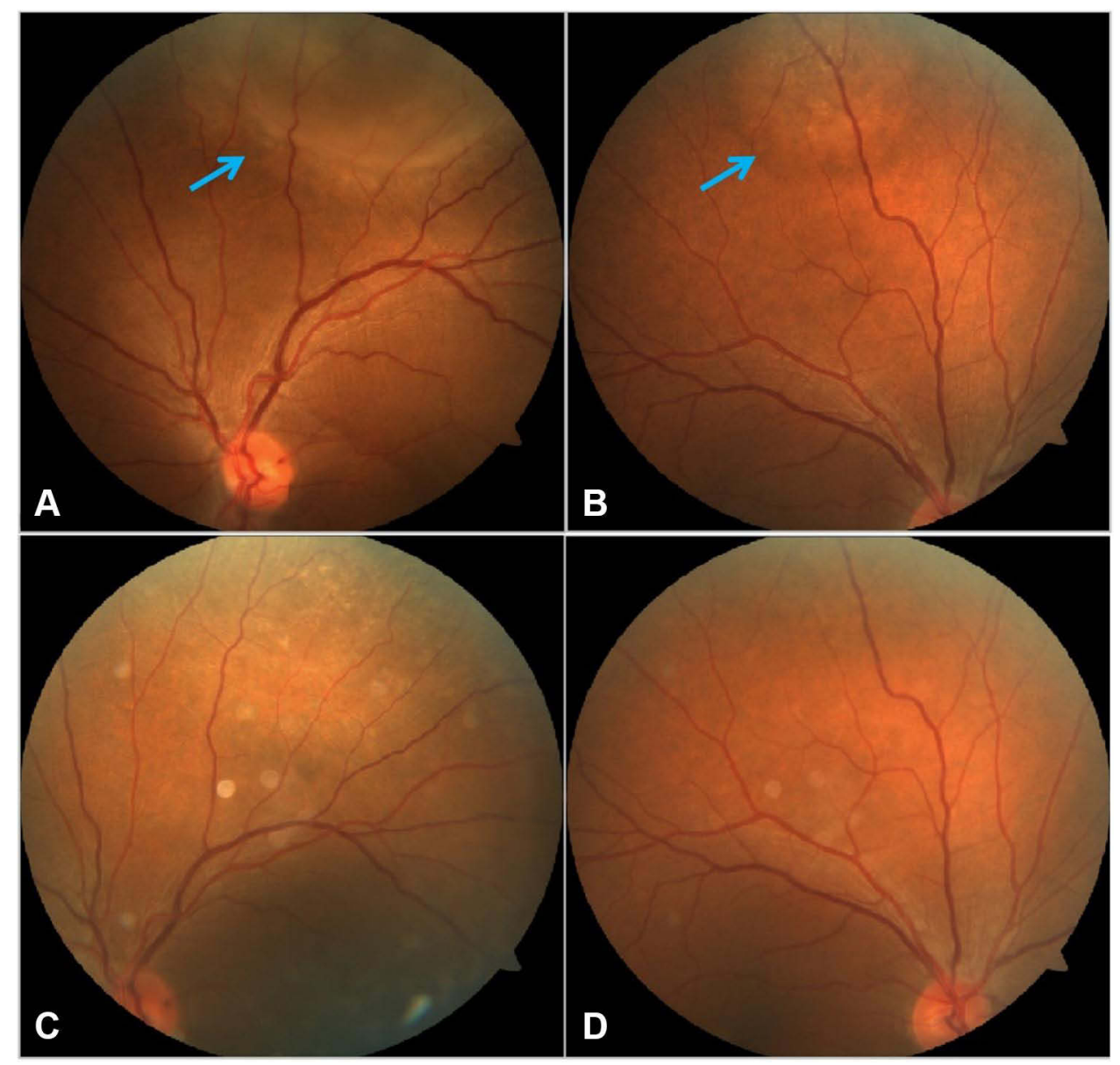

Figure 5 Dilated fundus examination of the 45-year-old male patient previously treated for breast cancer revealed a round relatively bump yellow-white choroid mass superior to the optic disc with serous leakage in each eye (left: (A); right: (B)). The lesions were annotated with blue arrows. Six months after being treated with fulvestrant and CDK4/6 inhibitor, the choroidal lesions regressed (left: (C); right: (D)).

Radiotherapy is another traditional treatment for CM, among which external beam radiotherapy (EBRT) is well known to all. ERBT helped nearly $80 \%$ female $\mathrm{BC}$ with $\mathrm{CM}$ cases reached regression of lesions in a prospective study. ${ }^{27}$ Hood et al described 3 cases of male BC metastasis to choroid and reviewed 19 cases that had been published earlier, of all the 22 male patients, 11 patients received EBRT with or without systemic therapies, and their choroid lesions regressed. ${ }^{28}$ Acute and late side effects of radiotherapy include erythema, conjunctivitis, cataract, keratopathy, retinopathy, neovascularization of the iris and optic neuropathy. Because metastatic cancers always lead to limited survival and short follow-up, both severe side effects and good tolerance of the radiotherapy were rarely reported in publications. ${ }^{29}$ Our patient therefore refused the radiotherapy.

The chief mechanism of CDK4/6 inhibitor to prevent tumor cell proliferation is inhibition of retinoblastoma protein phosphorylation and thus induction of cell cycle arrest from G1 to S phase. ${ }^{30}$ Data from PALOMA-3 trial indicated that in HR(+)/HER2(-) advanced BC, CDK4/6 inhibitor (palbociclib) in combination with fulvestrant led to a 6.9-month prolongation of OS. Moreover, subgroup analysis showed that in patients with sensitivity to previous endocrine therapy, palbociclib-fulvestrant could prolong the overall survival by 10 months than placebo-fulvestrant. In addition, among 311 patients with visceral metastases, palbociclib-fulvestrant group demonstrated a better OS compared to the control group 


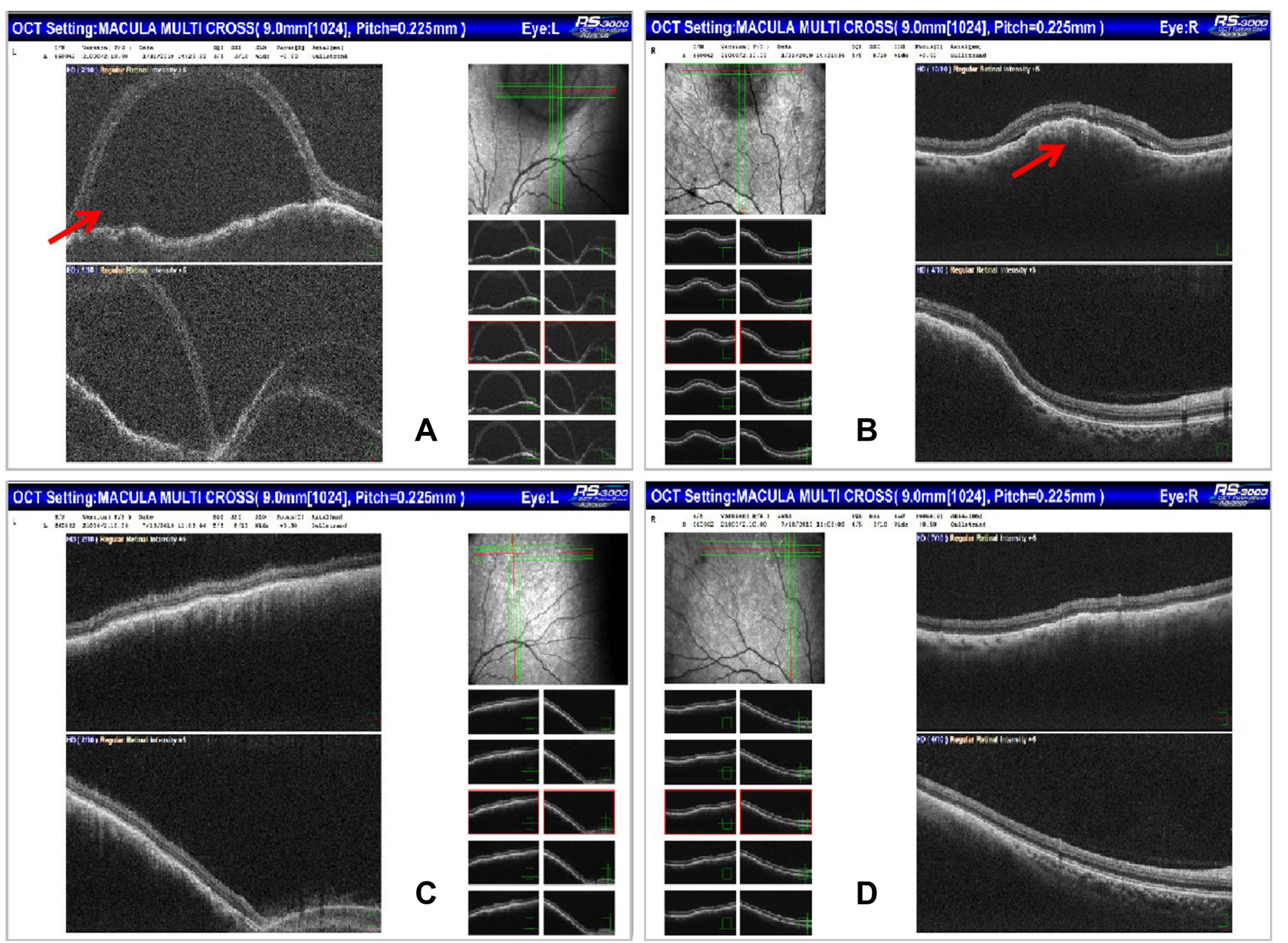

Figure 6 (A) OCT indicated limited detachment of the retinal neuroepithelial layer in the left eye, associated to SRF. (B) In the right eye, localized choroidal swelling was visible. The lesions were annotated with red arrows. After treatment of fulvestrant and CDK4/6 inhibitor, the SRF disappeared and retinas reattached (left: (C); right: (D)).

(27.6 months vs 24.7 months). ${ }^{31}$ Because our patient had already received adjuvant anastrozole as initial endocrine therapy for 5 years since 2012, when he got multiple systemic metastases in 2018, the first-line endocrine treatment of AI was believed to be failed. Meanwhile, according to the 2019 CSCO Breast Cancer Treatment and Treatment Guide, for HR(+)/HER2(-) patients who were refractory to initial AI therapy, CDK4/6 inhibitor plus fulvestrant was preferred. ${ }^{32}$ Therefore, based on PALOMA-3 trial data and 2019 CSCO breast cancer treatment guideline, the treatment strategy of palbociclib-fulvestrant was recommended to our patient.

Given the poor prognosis in $\mathrm{CM}$, doctors should be alert to the occurrence of ocular metastases in $\mathrm{BC}$ patients, both male and female, especially in those with visceral metastases. During the initial diagnosis and follow-up period, the eye examination can be selectively considered. Currently, the treatment strategy for BC with $\mathrm{CM}$ is still on debate. Yet, a combination of systemic and local therapy has reached consensus. To our knowledge, this is the first case where CDK4/ 6 inhibitor plus fulvestrant is used for male BC with CM and achieves a good response. The limitation of our study is the lack of histopathologic examination of the CM. Large prospective clinical trials need to be taken to confirm the effect of CDK4/6 inhibitor in CM. We hope the represented case can bring new idea on CM management to oncologists.

\section{Abbreviations}

$\mathrm{CM}$, choroidal metastasis; BC, breast cancer; CT, computed tomography; PET/CT, positron emission tomography/ computed tomography; IHC, Immunohistochemistry; ER, estrogen receptor; PR, progesterone receptor; HER2, human 


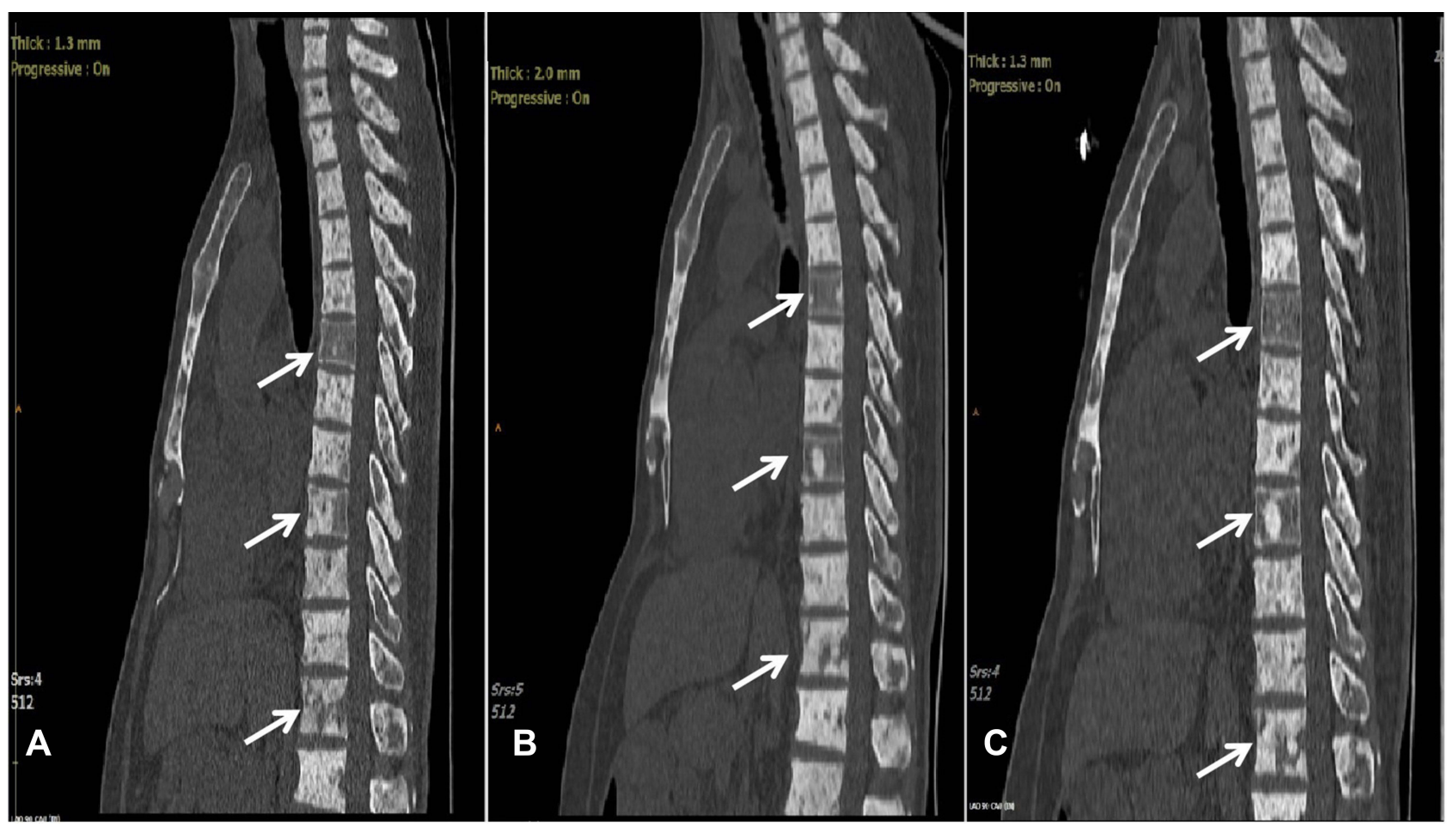

Figure 7 Spine CT showed the bone lesion remained stable before $(\mathbf{A})$ and after (B and $\mathbf{C})$ CDK4/6 inhibitor plus fulvestrant therapy. The lesions were annotated with white arrows.

epidermal growth factor receptor 2; OCT, optical coherence tomography; CDK4/6, cyclin-dependent kinases 4 and 6; SRF, subretinal fluid; AR, androgen receptor; OS, overall survival; IC-GA, indocyanine green fundus angiography; VEGF, vascular endothelial growth factor; HR, hormonal receptor; PDT, photodynamic therapy; ERBT, external beam radiotherapy; AI, aromatase-inhibitor.

\section{Data Sharing Statement}

The dataset used during the present study is available from the corresponding author upon a reasonable request.

\section{Ethics Approval and Consent to Participate}

The study was approved by the review board of the First Affiliated Hospital of Wenzhou Medical University. The patient has given written informed consent to publish his case, including publication of images.

\section{Consent to Publish}

Written informed consent to publication (including images, personal and clinical details of the participant) was obtained from the patient.

\section{Acknowledgments}

We would like to thank the Natural Science Foundation of Zhejiang Province of China (LY19H160026) and Zhejiang Provincial Health Department (2019KY454), whose financial support has made this study possible. In addition, we would like to thank Peng Li from the Department of Pathology and Huiru Liu from the Department of Radiology for helping us improve the case information. 


\section{Author Contributions}

All authors made a significant contribution to the work reported, whether that is in the conception, study design, execution, acquisition of data, analysis and interpretation, or in all these areas; took part in drafting, revising or critically reviewing the article; gave final approval of the version to be published; have agreed on the journal to which the article has been submitted; and agree to be accountable for all aspects of the work.

\section{Funding}

This work was funded by the Natural Science Foundation of Zhejiang Province of China (LY19H160026) and Zhejiang Provincial Health Department (2019KY454).

\section{Disclosure}

Weida Fu and Shurong Zheng are co-first authors of this study. The authors report no conflicts of interest in this work.

\section{References}

1. Giordano SH. Breast cancer in men. N Engl J Med. 2018;378:2311-2320. doi:10.1056/NEJMra1707939

2. Raj N, Padhy SK, Kumar V. Choroidal metastasis as the presenting feature of metastatic lung carcinoma. Clin Exp Optom. 2020;103:244-245. doi:10.1111/cxo. 12950

3. Shields CL, Welch RJ, Malik K, et al. Uveal metastasis: clinical features and survival outcome of 2214 tumors in 1111 patients based on primary tumor origin. Middle East Afr J Ophthalmol. 2018;25:81-90. doi:10.4103/meajo.MEAJO_6_18

4. Kreusel KM, Heimann H, Wiegel T, et al. Choroidal metastasis in men with metastatic breast cancer. Am J Ophthalmol. 1999;128(2):253-255. doi:10.1016/S0002-9394(99)00062-8

5. Wang X, Wang C, Guan J, et al. Progress of breast cancer basic research in China. Int J Biol Sci. 2021;17:2069-2079.

6. Sung H, Ferlay J, Siegel RL, et al. Global cancer statistics 2020: GLOBOCAN estimates of incidence and mortality worldwide for 36 cancers in 185 countries. CA Cancer J Clin. 2021;71:209-249. doi:10.3322/caac.21660

7. Gucalp A, Traina TA, Eisner JR, et al. Male breast cancer: a disease distinct from female breast cancer. Breast Cancer Res Treat. 2019;173:37-48.

8. Abdelwahab Yousef AJ. Male breast cancer: epidemiology and risk factors. Semin Oncol. 2017;44:267-272. doi:10.1053/j.seminoncol.2017.11.002

9. Pritzlaff M, Summerour P, McFarland R, et al. Male breast cancer in a multi-gene panel testing cohort: insights and unexpected results. Breast Cancer Res Treat. 2017;161:575-586. doi:10.1007/s10549-016-4085-4

10. Kwong A, Chau WW, Mang OW, et al. Male breast cancer: a population-based comparison with female breast cancer in Hong Kong, Southern China: 1997-2006. Ann Surg Oncol. 2014;21:1246-1253. doi:10.1245/s10434-013-3377-8

11. Giordano SH, Cohen DS, Buzdar AU, et al. Breast carcinoma in men: a population-based study. Cancer. 2004;101:51-57. doi:10.1002/cncr.20312

12. Wu Q, Li J, Zhu S, et al. Poorer breast cancer survival outcomes in males than females might be attributable to tumor subtype. Oncotarget. 2016;7:87532-87542. doi:10.18632/oncotarget.12052

13. Miao H, Verkooijen HM, Chia KS, et al. Incidence and outcome of male breast cancer: an international population-based study. $J$ Clin Oncol. 2011;29:4381-4386. doi:10.1200/JCO.2011.36.8902

14. Anderson WF, Jatoi I, Tse J, et al. Male breast cancer: a population-based comparison with female breast cancer. J Clin Oncol. 2010;28:232-239. doi:10.1200/JCO.2009.23.8162

15. Moore HCF. Breast cancer survivorship. Semin Oncol. 2020;47:222-228.

16. Tang J, Luo Y, Wu G. A glycolysis-related gene expression signature in predicting recurrence of breast cancer. Aging (Albany NY). 2020;12:24983-24994. doi:10.18632/aging.103806

17. Haidar YM, Korn BS, Rose MA. Complete regression of a choroidal metastasis secondary to breast cancer with stereotactic radiation: case report and review of literature. J Radiosurg SBRT. 2013;2:155-164.

18. Shields CL, Shields JA, Gross NE, et al. Survey of 520 eyes with uveal metastases. Ophthalmology. 1997;104:1265-1276. doi:10.1016/S01616420(97)30148-1

19. Mathis T, Jardel P, Loria O, et al. New concepts in the diagnosis and management of choroidal metastases. Prog Retin Eye Res. 2019;68:144-176.

20. Konstantinidis L, Rospond-Kubiak I, Zeolite I, et al. Management of patients with uveal metastases at the Liverpool Ocular Oncology Centre. $\mathrm{Br}$ J Ophthalmol. 2014;98:92-98. doi:10.1136/bjophthalmol-2013-303519

21. Al-Dahmash SA, Shields CL, Kaliki S, et al. Enhanced depth imaging optical coherence tomography of choroidal metastasis in 14 eyes. Retina. 2014;34:1588-1593. doi:10.1097/IAE.0000000000000131

22. Eide N, Walaas L. Fine-needle aspiration biopsy and other biopsies in suspected intraocular malignant disease: a review. Acta Ophthalmol. 2009;87:588-601. doi:10.1111/j.1755-3768.2009.01637.x

23. Arepalli S, Kaliki S, Shields CL. Choroidal metastases: origin, features, and therapy. Indian J Ophthalmol. 2015;63:122-127. doi:10.4103/03014738.154380

24. Parrozzani R, Frizziero L, Testi I, et al. Intraocular metastases secondary to breast carcinoma correlates with upregulation of estrogen and progesterone receptor expression in the primary tumor. Invest Ophthalmol Vis Sci. 2016;57:3944-3948. doi:10.1167/iovs.16-19695

25. Manquez ME, Shields CL, Karatza EC, et al. Regression of choroidal metastases from breast carcinoma using aromatase inhibitors. $\mathrm{Br}$ J Ophthalmol. 2005;89:776-777. doi:10.1136/bjo.2004.061127

26. Demirci H, Shields CL, Chao AN, et al. Uveal metastasis from breast cancer in 264 patients. Am J Ophthalmol. 2003;136:264-271.

27. Wiegel T, Bottke D, Kreusel KM, et al. External beam radiotherapy of choroidal metastases-final results of a prospective study of the German Cancer Society (ARO 95-08). Radiother Oncol. 2002;64:13-18. doi:10.1016/S0167-8140(02)00134-2 
28. Hood CT, Budd GT, Zakov ZN, et al. Male breast carcinoma metastatic to the choroid: report of 3 cases and review of the literature. Eur J Ophthalmol. 2011;21:459-467. doi:10.5301/EJO.2010.6191

29. Bajcsay A, Kontra G, Récsán Z, et al. Lens-sparing external beam radiotherapy of intraocular metastases: our experiences with twenty four eyes. Neoplasma. 2003;50:459-464.

30. Goel S, DeCristo MJ, McAllister SS, et al. CDK4/6 inhibition in cancer: beyond cell cycle arrest. Trends Cell Biol. 2018;28:911-925. doi:10.1016/j. tcb.2018.07.002

31. Turner NC, Slamon DJ, Ro J, et al. Overall survival with palbociclib and fulvestrant in advanced breast cancer. $N$ Engl $J$ Med. 2018;379:1926-1936. doi:10.1056/NEJMoa1810527

32. CSCO Breast Cancer Treatment Guide (Version 1. 2019) [Guideline]. Guidelines of Chinese society of clinical oncology (CSCO); 2019.

\section{Publish your work in this journal}

Cancer Management and Research is an international, peer-reviewed open access journal focusing on cancer research and the optimal use of preventative and integrated treatment interventions to achieve improved outcomes, enhanced survival and quality of life for the cancer patient. The manuscript management system is completely online and includes a very quick and fair peer-review system, which is all easy to use. Visit http://www.dovepress.com/testimonials.php to read real quotes from published authors.

Submit your manuscript here: https://www.dovepress.com/cancer-management-and-research-journa 